\title{
BOUNDS FOR THE BETTI NUMBERS OF GENERALIZED COHEN-MACAULAY IDEALS
}

\author{
LE TUAN HOA AND ROSA M. MIRÓ-ROIG
}

(Communicated by Wolmer V. Vasconcelos)

\begin{abstract}
Upper bounds for the Betti numbers of generalized Cohen-Macaulay ideals are given. In particular, for the case of non-degenerate, reduced and irreducible projective curves we get an upper bound which only depends on their degree.
\end{abstract}

\section{INTRODUCTION}

Let $I$ be a homogeneous ideal of a polynomial ring $S=\mathbf{K}\left[x_{1}, \ldots, x_{n}\right]$ over a field $\mathbf{K}, R=S / I, \underline{M}:=\left(x_{1}, \ldots, x_{n}\right), \underline{m}=\underline{M} R$ and $e=e(I):=e(R)$ the multiplicity of $R / I$. $I$ is said to have a property $P$ if $R$ has this property $P$.

It is a classical question to give upper and lower bounds for the Betti numbers, $\beta_{i}$, of $S / I$. A well-known conjecture due to Buchsbaum-Eisenbud says that $\beta_{i}(S / I) \geq\left(\begin{array}{l}n \\ i\end{array}\right)$ for 0 -dimensional ideals, and very recently Valla has given sharp upper bounds for the case of C.M. ideals (see [V]). The goal of this paper is to extend Valla's result to generalized C.M. ideals, i.e. ideals whose local cohomology modules $H_{\underline{m}}^{i}(R)$ are of finite length for all $i<\operatorname{dim}(R)$. As in [H], the key point is to reduce the computation to the case of C.M. ideals.

Now we give a brief description of the paper. In $\S 1$, we fix notations and recall some results needed later on. In $\S 2$, in order to prove our main result (Theorem 2.6), we first reduce to the case of 0-dimensional ideals and then we extend Valla's bounds to arbitrary (not necessarily non-degenerate) 0 -dimensional ideals. As a consequence and related to Buchsbaum-Eisenbud's conjecture we get the upper bound $\beta_{i}(S / I) \leq\left(\begin{array}{l}n \\ i\end{array}\right) e$ for the Betti numbers of any homogeneous 0 -dimensional ideal $I$. In the last section, applying our results we obtain upper bounds for the Betti numbers of the homogeneous ideal of some special projective schemes. In particular, for the case of non-degenerate, reduced and irreducible projective curves, $C$, we get an upper bound which only depends on the degree of the curve $C$.

Received by the editors September 30, 1993 and, in revised form, January 10, 1994.

1991 Mathematics Subject Classification. Primary 13D02.

The first author was supported by a grant of CRM, Institut d'Estudis Catalans. The second author was partially supported by DGICYT PB91-0231-C02-02. 


\section{NotATION AND PRELIMINARIES}

Throughout this paper we make the following conventions:

$$
\left(\begin{array}{c}
m \\
0
\end{array}\right)=1 \quad \text { if } m \geq 0, \quad \text { and } \quad\left(\begin{array}{l}
m \\
k
\end{array}\right)=0 \text { if } m<k .
$$

The following combinatorial formulae will be useful to us:

$$
\begin{gathered}
\left(\begin{array}{l}
n \\
i
\end{array}\right)+\cdots+\left(\begin{array}{c}
n-l \\
i
\end{array}\right)=\left(\begin{array}{c}
n+1 \\
i+1
\end{array}\right)-\left(\begin{array}{l}
n-l \\
i+1
\end{array}\right), \\
\sum_{i=a}^{b}\left(\begin{array}{l}
i \\
a
\end{array}\right)\left(\begin{array}{c}
c+i-1 \\
i
\end{array}\right)=\left(\begin{array}{c}
c+a-1 \\
a
\end{array}\right)\left(\begin{array}{l}
c+b \\
c+a
\end{array}\right), \\
\sum_{i=a}^{b} i\left(\begin{array}{l}
i \\
a
\end{array}\right)=-\left(\begin{array}{c}
b+2 \\
a+2
\end{array}\right)+(b+1)\left(\begin{array}{l}
b+1 \\
a+1
\end{array}\right) .
\end{gathered}
$$

If $m$ and $i$ are positive integers, then $m$ can be written uniquely in the form

$$
m=\left(\begin{array}{c}
m(i) \\
i
\end{array}\right)+\left(\begin{array}{c}
m(i-1) \\
i-1
\end{array}\right)+\cdots+\left(\begin{array}{c}
m(j) \\
j
\end{array}\right)
$$

where $m(i)>m(i-1)>\cdots>m(j) \geq j \geq 1$. This is called the $i$-binomial expansion of $m$. We let

$$
\begin{aligned}
& m^{\langle i\rangle}=\left(\begin{array}{c}
m(i)+1 \\
i+1
\end{array}\right)+\left(\begin{array}{c}
m(i-1)+1 \\
i
\end{array}\right)+\cdots+\left(\begin{array}{c}
m(j)+1 \\
j+1
\end{array}\right), \\
& m_{\langle i\rangle}=\left(\begin{array}{c}
m(i)-1 \\
i
\end{array}\right)+\left(\begin{array}{c}
m(i-1)-1 \\
i-1
\end{array}\right)+\cdots+\left(\begin{array}{c}
m(j)-1 \\
j
\end{array}\right)
\end{aligned}
$$

and $0^{\langle i\rangle}=0$. We define $r_{\langle t\rangle(0)}:=r$ and inductively $r_{\langle t\rangle(k)}=\left(r_{\langle t\rangle(k-1)}\right)_{\langle t\rangle}$.

If $I$ is a 0 -dimensional ideal we denote its Hilbert function by $H_{S / I}$.

(1.4) Following [ERV], $\S 4$, we denote by $J(e, h)$ the unique 0-dimensional lex-segment ideal in $S=\mathbf{K}\left[x_{1}, \ldots, x_{h}\right]$ with the Hilbert function $H_{S / J(e, h)}=$ $\left(1, h,\left(\begin{array}{c}h+1 \\ 2\end{array}\right), \ldots,\left(\begin{array}{c}h+t-2 \\ t-1\end{array}\right), r, 0, \ldots\right)$, where $t=t(e, h)$ is the unique integer such that $\left(\begin{array}{c}h+t-1 \\ t-1\end{array}\right) \leq e<\left(\begin{array}{c}h+t \\ t\end{array}\right)$ and $r=r(e, h)=e-\left(\begin{array}{c}h+t-1 \\ t-1\end{array}\right)$. We set $t(e, 0):=$ 1 .

(1.5) For $p=0, \ldots, h-1$, denote by $J_{p}(e, h)$ (resp. $\left.I_{p}\right)$ the image of $J(e, h)$ (resp. $I)$ in the polynomial ring $S_{p}:=\mathbf{K}\left[x_{1}, \ldots, x_{p}\right]$ under the canonical projection.

For short we also use the notation $e_{p}(e, h)=e\left(S_{p} / J_{p}(e, h)\right)$. In particular, $e_{0}(e, h)=1$. By [V], $e_{q}(e, n)=\left(\begin{array}{c}t-1+q \\ q\end{array}\right)+r_{\langle t\rangle(n-q)}$ for all $0 \leq q \leq n-1$.

(1.6) Let $H=(1, H(1), \ldots, H(a), \ldots)$ and $L=(1, L(1), \ldots, L(b), \ldots)$ be the Hilbert functions of two 0-dimensional homogeneous ideals of some polynomials rings, where $H(a) \neq 0$ and $L(b) \neq 0$. We write $H \cdot \geq L$ if $H(i) \geq L(i)$ for $i=0, \ldots, a-1$.

(1.7) By [ERV], Corollary 2.8, $H_{S_{h-1} / J_{h-1}(e, h)}(n)=\left(H_{S / J(e, h)}(n)\right)_{\langle n\rangle}$ for all $n \geq 1$.

The following lemma will be useful to us and it is essentially contained in the proof of [ERV], Theorem 3.10. 
Lemma 1.8. Assume that $1 \leq h \leq h^{\prime}$. Then, for all $p \geq 1$, we have $e_{h-p}(e, h) \leq$ $e_{h^{\prime}-p}\left(e, h^{\prime}\right)$.

Proof. Since $e(e, h-p)=0$ for $p \geq h$, we have only to consider the case $1 \leq$ $p \leq h-1$. Let $S=\mathbf{K}\left[x_{1}, \ldots, x_{h}\right]$ and $S^{\prime}=\mathbf{K}\left[x_{1}, \ldots, x_{h^{\prime}}\right]$. By the definition of $t$ and $t^{\prime}$ in (1.4) we have $t^{\prime}=t^{\prime}\left(e, h^{\prime}\right) \leq t=t(e, h)$. Hence, by (1.4) and (1.6), $H_{S^{\prime} / J\left(e, h^{\prime}\right)} \geq H_{S / J(e, h)}$. Moreover $e\left(S^{\prime} / J\left(e, h^{\prime}\right)\right)=e(S / J(e, h))=e$. By repeated application of [ERV], Lemma 3.9, and (1.7) we get $e_{h-p}(e, h) \leq$ $e_{h^{\prime}-p}\left(e, h^{\prime}\right)$, as required.

(1.9) (See [V], Proposition 2.) Let $I$ be a 0 -dimensional non-degenerated lex-segment ideal of $S$. Then, for every $i=1, \ldots, n$ we have

$$
\beta_{i}(S / I)=\sum_{p=i-1}^{n-1}\left(\begin{array}{c}
p \\
i-1
\end{array}\right) e\left(S_{p} / I_{p}\right)
$$

(1.10) For the basic properties of Buchsbaum as well as generalized C.M. rings we refer the reader to the book [SV]. A ring $R$ is called a generalized C.M. ring if the length of the local cohomology modules $H_{\mathfrak{m}}^{i}(R)$ is finite for all $i<\operatorname{dim}(R)$. In this case we set $I(R):=\bigoplus_{i=0}^{d-1}\left(\begin{array}{c}d-1 \\ i\end{array}\right) l\left(H_{\mathfrak{m}}^{i}(R)\right) . \quad R$ is a generalized C.M. ring if and only if there exists a positive integer $k$ such that $\mathrm{m}^{k}$ is an $R$-standard ideal. $R$ is called a Buchsbaum ring if $\mathrm{m}$ is an $R$-standard ideal.

\section{MAIN Results}

All results of $\S 2$ also hold for ideals of a regular local ring. For the simplicity of formulation we restrict our attention to the case of homogeneous ideals in a polynomial ring.

Recall that a homogeneous element $x \in \mathfrak{m}$ is called a superficial element of order 1 for $\mathfrak{m}$ if there exists a positive integer $p$ such that $\left(\mathfrak{m}^{q}: x\right) \cap \mathfrak{m}^{p}=\mathfrak{m}^{q-1}$ for $q>>0$. For the properties of superficial elements see, e.g., [S].

Lemma 2.1. If $x \in \mathfrak{m}$ is a superficial element of order 1 for $\mathfrak{m}$, then its image in $R /(0: x)$ is a superficial element of order 1 for $\mathfrak{m} /(0: x)$.

Let $I \subset S=\mathbf{K}\left[x_{1}, \ldots, x_{n}\right]$ be a homogeneous ideal. Set $R=S / I$.

Lemma 2.2. Assume that $I$ is a generalized C.M. ideal and $\operatorname{dim}(R)=d$. Let $\delta=\operatorname{depth}(R)$. Then, for all $1 \leq i \leq n$ we have

$$
\beta_{i}(S / I) \leq\left(\left(\begin{array}{c}
n-\delta+1 \\
i+1
\end{array}\right)-\left(\begin{array}{c}
n-d+1 \\
i+1
\end{array}\right)\right) I(R)+\beta_{i}\left(S^{\prime} / J\right),
$$

where $J$ is a 0 -dimensional ideal of a polynomial ring $S^{\prime}=\mathbf{K}\left[y_{1}, \ldots, y_{n-d}\right]$ with $e(J)=e(I)$.

Proof. Assume that $\delta=0$. Let $x$ (resp. the image of $x$ ) be a homogeneous superficial element of order 1 for $\mathfrak{M}$ (resp. for $\mathfrak{m}$ ) (see [S], p. 7, for the existence of $x$ ). Consider the exact sequence

$$
0 \rightarrow(0: x) \rightarrow R \rightarrow R /(0: x) \rightarrow 0 .
$$

Note that $l((0: x)) \leq l\left(H_{\mathrm{m}}^{0}(R)\right) \leq I(R)$. Applying the functor $\operatorname{Tor} S_{(\mathbf{K}, \cdot)}$ to 
the above exact sequence we get

$$
\begin{aligned}
\beta_{i}(S / I) & =\operatorname{dim} \operatorname{Tor}_{i}^{S}(\mathbf{K}, R) \leq \operatorname{dim} \operatorname{Tor}_{i}^{S}(\mathbf{K},(0: x))+\operatorname{dim} \operatorname{Tor}_{i}^{S}(\mathbf{K}, R /(0: x)) \\
& \leq\left(\begin{array}{c}
n \\
i
\end{array}\right) I(R)+\operatorname{dim} \operatorname{Tor}_{i}^{S}(\mathbf{K}, R /(0: x)) .
\end{aligned}
$$

By [HSV], Lemma 1,

$$
\operatorname{Tor}_{i}(\mathbf{K}, R /(0: x)) \cong \operatorname{Tor}_{i}^{S_{1}}(\mathbf{K}, R /(0: x+x R))=\beta_{i}\left(S_{1} / J_{1}\right),
$$

where $S_{1}:=S /(x) J_{1}=(I: x)+(x) /(x) \subset S_{1}$ is again a polynomial ring and $J_{1}$ is a homogeneous ideal. By Lemma 2.1, the image of $x$ is a superficial element of order 1 for $R /(0: x)$. Moreover, the image of $x$ is a non-zero divisor of $R /(0: x)$. Hence, $e\left(J_{1}\right)=e(R /(0: x))=e(I)$. Repeating this process we get

$$
\begin{aligned}
\beta_{i}(S / I) & \leq\left(\left(\begin{array}{c}
n \\
i
\end{array}\right)+\cdots+\left(\begin{array}{c}
n-d+1 \\
i
\end{array}\right)\right) I(R)+\beta_{i}\left(S^{\prime} / J\right) \\
& =\left(\left(\begin{array}{c}
n+1 \\
i+1
\end{array}\right)-\left(\begin{array}{c}
n-d+1 \\
i+1
\end{array}\right)\right) I(R)+\beta_{i}\left(S^{\prime} / J\right),
\end{aligned}
$$

where $J$ is a 0 -dimensional ideal of a polynomial ring $S^{\prime}=\mathbf{K}\left[y_{1}, \ldots, y_{n-d}\right]$ with $e(J)=e(I)$.

If $\delta>0$, then in the first $\delta$ steps we have $\operatorname{Tor}_{i}^{S}(\mathbf{K}, 0: x)=0$, and the result easily follows.

Let $\beta_{i}(e, n)=\beta_{i}(S / J(e, n))$ be Valla's bound for the $i$ th Betti number of non-degenerate 0 -dimensional ideals in $\mathbf{K}\left[x_{1}, \ldots, x_{n}\right]$ with multiplicity $e$ ([V], Theorem 4). We set $\beta_{i}(e, n)=1$ if $i=0$ and $\beta_{i}(e, n)=0$ if $i<0$. We will extend Valla's results to any 0 -dimensional ideal.

Lemma 2.3. Let $I \subset \mathbf{K}\left[x_{1}, \ldots, x_{n}\right]$ be a 0 -dimensional ideal of multiplicity $e(I)=e$. Set $l=\operatorname{dim}_{\mathbf{K}}\left(I \backslash \mathfrak{M}^{2}\right) \otimes \mathbf{K}$. Then, for all $i=1, \ldots, n$, we have

$$
\beta_{i}(S / I) \leq \sum_{j=0}^{l}\left(\begin{array}{l}
l \\
j
\end{array}\right) \beta_{i-j}(e, n-l) .
$$

Proof. We proceed by induction on $l$. For $l=0$ it follows from (1.9). Assume $l>0$; let $x \in I \backslash \mathfrak{M}^{2}$ be any linear form. By changing the coordinates we can assume that $x=x_{n}$. Let $I^{\prime}=I / x S, S^{\prime}=S / x S \cong \mathbf{K}\left[x_{1}, \ldots, x_{n-1}\right]$. Then $\operatorname{dim}\left(I^{\prime} \backslash \mathfrak{M}^{\prime 2}\right) \otimes \mathbf{K}=l-1$ and $e\left(I^{\prime}\right)=e(I)$. By [HSV], Lemma 1 and the induction hypothesis we have the following required result:

$$
\begin{aligned}
\beta_{i}(S / I) \leq & \beta_{i-1}\left(S^{\prime} / I^{\prime}\right)+\beta_{i}\left(S^{\prime} / I^{\prime}\right) \\
\leq & \sum_{j=0}^{l-1}\left(\begin{array}{c}
l-1 \\
j
\end{array}\right) \beta_{i-1-j}(e,(n-1)-(l-1)) \\
& +\sum_{j=0}^{l-1}\left(\begin{array}{c}
l-1 \\
j
\end{array}\right) \beta_{i-j}(e,(n-1)-(l-1)) \\
= & \beta_{i-l}(e, n-l)+\sum_{j=1}^{l-1}\left(\left(\begin{array}{c}
l-1 \\
j-1
\end{array}\right)+\left(\begin{array}{c}
l-1 \\
j
\end{array}\right)\right) \beta_{i-j}(e, n-l)+\beta_{i}(e, n) \\
= & \sum_{j=0}^{l}\left(\begin{array}{l}
l \\
j
\end{array}\right) \beta_{i-j}(e, n-l) .
\end{aligned}
$$


Lemma 2.4. Let $I$ be a 0-dimensional ideal of $S$ of multiplicity $e$. Denote $n^{*}=\min (e-1, n)$, and define $t^{*}, r^{*}$ after the formulae in (1.4) for $e$ and $n^{*}$. Then, for all $i=1, \ldots, n$, we have

$$
\beta_{i}(S / I) \leq\left(\begin{array}{c}
t^{*}+i-2 \\
t^{*}-1
\end{array}\right)\left(\begin{array}{c}
t^{*}+n-1 \\
t^{*}+i-1
\end{array}\right)+\sum_{p=i-1}^{n-1}\left(\begin{array}{c}
p \\
i-1
\end{array}\right) r_{\left\langle i^{*}\right\rangle(n-p)}^{*} .
$$

Proof. Set $l=\operatorname{dim}_{K}\left(I \backslash \mathfrak{M}^{2}\right) \otimes \mathbf{K}$. If $n=l$, then $I=\left(x_{1}, \ldots, x_{n}\right)$ and the above formula is trivially true. Assume $n-l \geq 1$. By Lemma 2.3 and (1.9) we have

$$
\begin{aligned}
\beta_{i}(S / I) & \leq \sum_{j=0}^{l}\left(\begin{array}{l}
l \\
j
\end{array}\right) \beta_{i-j}(e, n-l)=\sum_{j=0}^{\min (i-1, l)}\left(\begin{array}{l}
l \\
j
\end{array}\right) \beta_{i-j}(e, n-l)+\left(\begin{array}{l}
l \\
i
\end{array}\right) \\
& =\left(\begin{array}{l}
l \\
i
\end{array}\right)+\sum_{j=0}^{\min (i-1, l)}\left(\begin{array}{l}
l \\
j
\end{array}\right) \sum_{p=i-j-l}^{n-l-1}\left(\begin{array}{c}
p \\
i-j-1
\end{array}\right) e_{p}(e, n-l) \\
& =\left(\begin{array}{l}
l \\
i
\end{array}\right)+\sum_{p=i-\min (i-1, l)-1}^{n-l-1} e_{p}(e, n-l) \sum_{j=i-p-1}^{\min (i-1, l)}\left(\begin{array}{l}
l \\
j
\end{array}\right)\left(\begin{array}{c}
p \\
i-j-1
\end{array}\right) .
\end{aligned}
$$

Since $\sum_{j=i-p-1}^{\min (i-1, l)}\left(\begin{array}{l}l \\ j\end{array}\right)\left(\begin{array}{c}p \\ i-j-1\end{array}\right) \leq\left(\begin{array}{c}p+l \\ i-1\end{array}\right)$ and $e_{p}=0$ for $p<0$, by Lemma 1.8 we have

$$
\begin{aligned}
\beta_{i}(S / I) & \leq\left(\begin{array}{l}
l \\
i
\end{array}\right)+\sum_{p=0}^{n-l-1}\left(\begin{array}{c}
p+l \\
i-1
\end{array}\right) e_{p}(e, n-l) \\
& \leq\left(\begin{array}{l}
l \\
i
\end{array}\right)+\sum_{p=0}^{n-l-1}\left(\begin{array}{c}
p+l \\
i-1
\end{array}\right) e_{n^{*}-(n-l)+p}\left(e, n^{*}\right) \\
& =\left(\begin{array}{l}
l \\
i
\end{array}\right)+\sum_{q=l}^{n-1}\left(\begin{array}{c}
q \\
i-1
\end{array}\right) e_{n^{*}-n+q}\left(e, n^{*}\right) \\
& =\left(\begin{array}{l}
l \\
i
\end{array}\right)+\sum_{q=\max (l, i-1)}^{n-1}\left(\begin{array}{c}
q \\
i-1
\end{array}\right) e_{n^{*}-n+q}\left(e, n^{*}\right) .
\end{aligned}
$$

Hence, using (1.5), we get

$$
\begin{aligned}
\beta_{i}(S / I) \leq & \left(\begin{array}{l}
l \\
i
\end{array}\right)+\sum_{q=\max (l, i-1)}^{n-1}\left(\begin{array}{c}
q \\
i-1
\end{array}\right)\left(\begin{array}{c}
t^{*}-1+n^{*}-n+q \\
t^{*}-1
\end{array}\right) \\
& +\sum_{q=\max (l, i-1)}^{n-1}\left(\begin{array}{c}
q \\
i-1
\end{array}\right) r_{\left\langle t^{*}\right)(n-q)}^{*} \\
\leq & \left(\begin{array}{l}
l \\
i
\end{array}\right)+\sum_{q=\max (l, i-1)}^{n-1}\left(\begin{array}{c}
q \\
i-1
\end{array}\right)\left(\begin{array}{c}
t^{*}-1+q \\
t^{*}-1
\end{array}\right)+\sum_{q=i-1}^{n-1}\left(\begin{array}{c}
q \\
i-1
\end{array}\right) r_{\left.l t^{*}\right\rangle(n-q)}^{*} .
\end{aligned}
$$


If $l<i,\left(\begin{array}{l}l \\ i\end{array}\right)=0$, and if $l \geq i$, we have

$$
\sum_{q=i-1}^{l-1}\left(\begin{array}{c}
q \\
i-1
\end{array}\right)\left(\begin{array}{c}
t^{*}-1+q \\
t^{*}-1
\end{array}\right) \geq \sum_{q=i-1}^{l-1}\left(\begin{array}{c}
q \\
i-1
\end{array}\right)=\left(\begin{array}{l}
l \\
i
\end{array}\right) .
$$

So in both cases we obtain

$$
\begin{aligned}
\left(\begin{array}{l}
l \\
i
\end{array}\right)+\sum_{q=\max (l, i-1)}^{n-1}\left(\begin{array}{c}
q \\
i-1
\end{array}\right)\left(\begin{array}{c}
t^{*}-1+q \\
t^{*}-1
\end{array}\right) & \leq \sum_{q=i-1}^{n-1}\left(\begin{array}{c}
q \\
i-1
\end{array}\right)\left(\begin{array}{c}
t^{*}-1+q \\
q
\end{array}\right) \\
& =\left(\begin{array}{c}
t^{*}+i-2 \\
t^{*}-1
\end{array}\right)\left(\begin{array}{c}
t^{*}+n-1 \\
t^{*}+i-1
\end{array}\right),
\end{aligned}
$$

which completes the proof of the lemma.

Remark 2.4.1. If $e-1 \geq n$, then $n=n^{*}$ and we get exactly the same bound as Valla's bound for non-degenerate ideals ([V], Proposition 5(i)). Hence, we cannot improve Lemma 2.4 unless one involves $l$. But $l$ is not defined explicitly in our consideration.

Remark 2.4.2. Since $e_{p}\left(e, n^{*}\right)=\operatorname{length}\left(S_{p} / J_{p}\left(e, n^{*}\right)\right) \leq e-\left(n^{*}-p\right)$ for all $0 \leq p \leq n^{*}-1$, from the proof of Lemma 2.4 we get another estimation:

$$
\beta_{i}(S / I) \leq \sum_{q=i-1}^{n-1}\left(\begin{array}{c}
q \\
i-1
\end{array}\right) e=\left(\begin{array}{c}
n \\
i
\end{array}\right) e .
$$

For $n=n^{*}$ we get a little more:

$$
\begin{aligned}
\beta_{i}(S / I) & \leq \sum_{q=i-1}^{n-1}\left(\begin{array}{c}
q \\
i-1
\end{array}\right)(e-(n-q))=\left(\begin{array}{c}
n \\
i
\end{array}\right)(e-n)+\sum_{q=i-1}^{n-1} q\left(\begin{array}{c}
q \\
i-1
\end{array}\right) \\
& =\left(\begin{array}{c}
n \\
i
\end{array}\right)(e-n)-\left(\begin{array}{c}
n+1 \\
i+1
\end{array}\right)+n\left(\begin{array}{c}
n \\
i
\end{array}\right)=\left(\begin{array}{c}
n \\
i
\end{array}\right) e-\left(\begin{array}{c}
n+1 \\
i+1
\end{array}\right) .
\end{aligned}
$$

It is interesting to compare this last result with the conjecture that $\beta_{i}(S / I) \geq$ $\left(\begin{array}{c}n \\ i\end{array}\right)$.

Lemma 2.5. $r_{\langle t\rangle(n-q)} \leq\left(\begin{array}{c}t+q-1 \\ t\end{array}\right)$ for all $0 \leq q \leq n$.

From Lemma 2.2 and Lemma 2.4 we get

Theorem 2.6. Assume that $I$ is a generalized C.M. ideal of $\mathrm{ht}(I)=h$ in $\mathbf{K}\left[x_{1}, \ldots, x_{n}\right] ; \delta=\operatorname{depth}(R)$. Let $h^{*}=\min (e-1, h)$ and $t^{*}, r^{*}$ be defined for $e$ and $h^{*}$. Then for all $1 \leq i \leq n$ we have

$$
\begin{aligned}
\beta_{i}(S / I) \leq & \left(\begin{array}{c}
n+1-\delta \\
i+1
\end{array}\right)-\left(\begin{array}{c}
h+1 \\
i+1
\end{array}\right) I(R) \\
& +\left(\begin{array}{c}
t^{*}+i-2 \\
t^{*}-1
\end{array}\right)\left(\begin{array}{c}
t^{*}+h-1 \\
t^{*}+i-1
\end{array}\right)+\sum_{p=i-1}^{h-1}\left(\begin{array}{c}
p \\
i-1
\end{array}\right) r_{\left\langle t^{*}\right\rangle(h-p)}^{*} \\
\leq & \left(\left(\begin{array}{c}
n+1-\delta \\
i+1
\end{array}\right)-\left(\begin{array}{c}
h+1 \\
i+1
\end{array}\right)\right) I(R)+\left(\begin{array}{c}
t^{*}+i-1 \\
t^{*}
\end{array}\right)\left(\begin{array}{c}
t^{*}+h \\
t^{*}+i
\end{array}\right) .
\end{aligned}
$$


Proof. The first inequality follows immediately from Lemma 2.2 and Lemma 2.4. Further, by Lemma 2.5 we have

$$
\begin{aligned}
\sum_{p=i-1}^{h-1}\left(\begin{array}{c}
p \\
i-1
\end{array}\right) \delta_{\left\langle t^{*}\right\rangle(h-p)}^{*} & \leq \sum_{p=i-1}^{h-1}\left(\begin{array}{c}
p \\
i-1
\end{array}\right)\left(\begin{array}{c}
t^{*}+p+h^{*}-h-1 \\
t^{*}
\end{array}\right) \\
& \leq \sum_{p=i-1}^{h-1}\left(\begin{array}{c}
p \\
i-1
\end{array}\right)\left(\begin{array}{c}
t^{*}+p-1 \\
t^{*}
\end{array}\right) \\
& =\sum_{p=i-1}^{h-1}\left(\begin{array}{c}
p \\
i-1
\end{array}\right)\left(\left(\begin{array}{c}
t^{*}+p \\
p
\end{array}\right)-\left(\begin{array}{c}
t^{*}+p-1 \\
p
\end{array}\right)\right) \\
& =\left(\begin{array}{c}
t^{*}+i-1 \\
t^{*}
\end{array}\right)\left(\begin{array}{c}
t^{*}+h \\
t^{*}+i
\end{array}\right)-\left(\begin{array}{c}
t^{*}+i-2 \\
t^{*}-1
\end{array}\right)\left(\begin{array}{c}
t^{*}+h-1 \\
t^{*}+i-1
\end{array}\right) .
\end{aligned}
$$

From that we get the second inequality.

Remark 2.7. Other bounds are (by Remarks 2.4.1 and 2.4.2)

$$
\beta_{i}(S / I) \leq\left(\left(\begin{array}{c}
n+1-\delta \\
i+1
\end{array}\right)-\left(\begin{array}{c}
h+1 \\
i+1
\end{array}\right)\right) I(R)+\left(\begin{array}{c}
h \\
i
\end{array}\right) e,
$$

or for $e \geq n-1$

$$
\beta_{i}(S / I) \leq\left(\left(\begin{array}{c}
n+1-\delta \\
i+1
\end{array}\right)-\left(\begin{array}{c}
h+1 \\
i+1
\end{array}\right)\right) I(R)+\left(\begin{array}{c}
h \\
i
\end{array}\right) e-\left(\begin{array}{c}
h+1 \\
i+1
\end{array}\right) .
$$

Example. For $I=\left(x_{1}, \ldots, x_{n-1}\right) \cap\left(x_{1}, \ldots, x_{n}\right)^{2}$ we have $I(R)=n-1$, $t^{*}=1, r^{*}=0$. Hence, the first bound in Theorem 2.6 equals the bound in Remark 2.7 and equals $\left(\begin{array}{c}n \\ i\end{array}\right)(n-1)+\left(\begin{array}{c}n-1 \\ i\end{array}\right)=\left(\begin{array}{c}n \\ i\end{array}\right)((n-1)+(i+1)(n-i) / n)$. Using [EK] we get

$\beta_{i}(S / I)=\left(\begin{array}{c}n \\ i\end{array}\right)(n-1)+\left(\begin{array}{c}n-1 \\ i+1\end{array}\right)=\left(\begin{array}{c}n \\ i\end{array}\right)((n-1)+(n-i)(n-i-1) /((i+1) n))$.

This shows that in this example the bounds given in Theorem 2.6 are not far of being sharp.

In order to get bounds for the Betti numbers in terms of $e, n$ and $k$ where $k$ is defined in (1.10) one can combine Theorem 2.6 with the following bounds on $I(R)$ given by the first author in $[\mathrm{H}]$, where one can also find better bounds for $\beta_{1}(S / I)$. Note that there is already no upper bound for the number of generators of $I$ which does not involve $k$. Following [EVR], $\S 4$, we set $\nu(e, h)=\left(\begin{array}{c}h+t-1 \\ t\end{array}\right)-$ $r+r^{\langle t\rangle}$ where $t$ and $r$ are defined in (14).

Lemma 2.8. Let $I$ be an ideal of $S$ such that $\mathrm{m}^{k}$ is an $R$-standard ideal $(k>$ 0). Then

(i) $I(R) \leq\left(n-1-n^{\prime}+\nu\left(k^{d-1} e, n^{\prime}\right)\right)\left(\begin{array}{c}n+k-1 \\ k-1\end{array}\right)$, where

$$
n^{\prime}=\min \left(n-1, k^{d-1} e-1\right) \text {. }
$$

(ii) $I(R) \leq\left((2 n-3) /(n-1)+e k^{d-1}(n-2)^{2} /(n-1)\right)\left(\begin{array}{c}n+k-1 \\ k-1\end{array}\right)$.

(iii) If $\operatorname{depth}(R)>0$, then $I(R) \leq(d-1)\left(k^{d} e-1\right)$.

(iv) If $I$ is a Buchsbaum ideal, then $I(R) \leq h-h^{\prime}+\nu\left(e, h^{\prime}\right)$, where $h^{\prime}=$ $\min (h, e-1)$. 


\section{ApPLications}

As applications of our results we will give upper bounds for the Betti numbers of the homogeneous ideal of some projective schemes.

Example 3.1. Let $V$ be an arithmetically Buchsbaum projective subscheme of codimension 2 in $\mathbf{P}^{n}$. Then by [H], Corollary $4.1, I(R) \leq(1+8 e)^{1 / 2}$ and $t^{*} \leq\left(-1+(1+8 e)^{1 / 2}\right) / 2$. Hence by Theorem 2.6 we get

$$
\beta_{i}\left(S / I_{V}\right) \leq\left(\left(\begin{array}{c}
n+1 \\
i+1
\end{array}\right)-\left(\begin{array}{c}
3 \\
i+1
\end{array}\right)\right)(1+8 e)^{1 / 2}+\left(\begin{array}{c}
t^{*}+i-1 \\
t^{*}
\end{array}\right)\left(\begin{array}{c}
t^{*}+2 \\
t^{*}+i
\end{array}\right) .
$$

Thus:

(1) $\beta_{1}\left(S / I_{V}\right) \leq\left(\left(\begin{array}{c}n+1 \\ 2\end{array}\right)-3\right)(1+8 e)^{1 / 2}+\left(3+(1+8 e)^{1 / 2}\right) / 2=\left(\begin{array}{c}n+1 \\ 2\end{array}\right)(1+8 e)^{1 / 2}+$ $3 / 2-5(1+8 e)^{1 / 2} / 2$.

(2) $\beta_{2}\left(S / I_{V}\right) \leq\left(\left(\begin{array}{c}n+1 \\ 3\end{array}\right)-1\right)(1+8 e)^{1 / 2}+\left(1+(1+8 e)^{1 / 2}\right) / 2=\left(\begin{array}{c}n+1 \\ 3\end{array}\right)(1+8 e)^{1 / 2}+$ $1 / 2-\left((1+8 e)^{1 / 2}\right) / 2$.

(3) For all $n \geq i \geq 3, \beta_{i}\left(S / I_{V}\right) \leq\left(\begin{array}{c}n+1 \\ i+1\end{array}\right)(1+8 e)^{1 / 2}$.

Example 3.2. Let $V$ be an arithmetically Buchsbaum projective subscheme of codimension $h$ in $\mathbf{P}^{n}$. Then, we have the following bounds on the Betti numbers:

$$
\beta_{i}\left(S / I_{V}\right) \leq\left(\left(\begin{array}{c}
n+2-\delta \\
i+1
\end{array}\right)-\left(\begin{array}{c}
h+1 \\
i+1
\end{array}\right)\right)(d-1)(e-1)+\left(\begin{array}{c}
h \\
i
\end{array}\right) e-\left(\begin{array}{c}
h+1 \\
1+i
\end{array}\right) .
$$

In particular, if $d=2$ (i.e., for curves)

$$
\beta_{i}\left(S / I_{V}\right) \leq\left(\begin{array}{c}
n \\
i
\end{array}\right)(e-1)+\left(\begin{array}{c}
n-1 \\
i
\end{array}\right) e-\left(\begin{array}{c}
n \\
1+i
\end{array}\right)
$$

Example 3.3. Let $C$ be a non-degenerate, reduced and irreducible curve in $\mathbf{P}^{n}$ over an algebraically closed field $\mathbf{K}$. From Theorem 2.6 and Remark 2.7 we get

$$
\beta_{i}\left(S / I_{C}\right) \leq\left(\begin{array}{c}
n \\
i
\end{array}\right) I(R)+\left(\begin{array}{c}
n-1 \\
i
\end{array}\right) e-\left(\begin{array}{c}
n \\
i+1
\end{array}\right) .
$$

Using Lemma 2.8 (iii) we obtain

$$
\beta_{i}\left(S / I_{C}\right) \leq\left(\begin{array}{c}
n \\
i
\end{array}\right)\left(k^{2} e-1\right)+\left(\begin{array}{c}
n-1 \\
i
\end{array}\right) e-\left(\begin{array}{c}
n \\
i+1
\end{array}\right) .
$$

By [GLP], one can choose $k \leq \max (1, e-n)$; hence if $e \geq n+1$ we have

$$
\beta_{i}\left(S / I_{C}\right) \leq\left(\begin{array}{c}
n \\
i
\end{array}\right)\left((e-n)^{2} e-1\right)+\left(\begin{array}{c}
n-1 \\
i
\end{array}\right) e-\left(\begin{array}{c}
n \\
i+1
\end{array}\right),
$$

which only depends on $e$.

\section{REFERENCES}

[EH] S. Eliahou and M. Kervaire, Minimal resolution of some monomial ideals, J. Algebra 129 (1990), 1-25.

[ERV] J. Elias, L. Robbiano, and G. Valla, Number of generators of ideals, Nagoya Math. J. 123 (1991), 39-76.

[GLP] L. Gruson, C. Peskine, and R. Lazarsfeld, On a theorem of Castelnuovo and the equations defining space curves, Invent. Math. 72 (1983), 491-506. 
[H] L. T. Hoa, Bounds for the number of generators of generalized Cohen-Macaulay ideals, preprint, 1993.

[HSV] L. T. Hoa, J. Stückrad, and W. Vogel, Towards a structure theory for projective varieties of degree=codimension +2, J. Pure Appl. Algebra 71 (1991), 203-231.

[S] J. Sally, Number of generators in ideals in local rings, Lecture Notes in Pure and Appl. Math., vol. 35, Dekker, New York, 1978.

[SV] J. Stückrad and W. Vogel, Buchsbaum rings and applications, Springer, Berlin and Heidelberg, 1986.

[V] G. Valla, On the Betti numbers of perfect ideals, Compositio Math. 91 (1994), 305-320.

Institute of Mathematics, P. O. Box 631 Bo ho, Hanol, Vietnam

Departamento Algebra y Geometria, facultad de Matematicas, Universidad de Barcelona, 08007 Barcelona, SPAIN

E-mail address: miroccerber.ub.es 